\title{
Thermal / Visible Autonomous Stereo Visio System Calibration Methodology for Non-controlled Environments
}

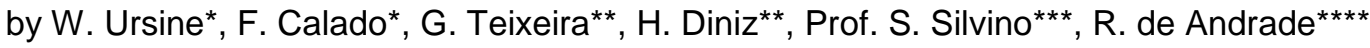

\begin{abstract}
*Postgraduate Program in Electrical Engineering - Universidade Federal de Minas Gerais - Av. Antônio Carlos, 6627, 31270-901, Belo Horizonte, MG, Brazil, wagnerursine@gmail.com, flavio.calado@ig.com.br

${ }^{* * P}$ ostgraduate Program in Mechanical Engineering - Universidade Federal de Minas Gerais - Av. Antônio Carlos, 6627, 31270-901, Belo Horizonte, MG, Brazil, guiemail@gmail.com,HENRIQUE.DINIZ@cemig.com.br

${ }^{* * *}$ Postgraduate Program in Electrical Engineering - Universidade Federal de Minas Gerais - Av. Antônio Carlos, 6627, 31270-901, Belo Horizonte, MG, Brazil,silvino@cpdee.ufmg.br

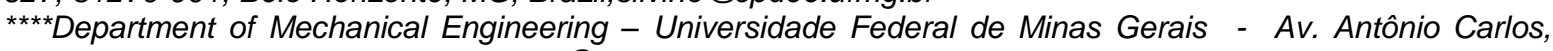
6627, 31270-901, Belo Horizonte, MG, Brazil,rma@ufmg.br
\end{abstract}

\section{Abstract}

This paper presents a fast and low cost methodology for thermal / visible stereo vision system calibration with high reliability for non-controlled external environments. The proposed methodology uses a non-powered calibration rig to map the coordinates, field of view and magnification distortions from visible and thermal vision and a self-calibration analysis procedure to estimate metrological uncertainty of imaging correspondence. Tests were conducted to determine the effectiveness of the developed calibration rig.

\section{Introduction}

Hybrid Systems, formed by the combination of visible and thermal sensors, have been widely adopted in applications related to inspection and monitoring. Thermal inspection increases the reliability of some equipment, since it is able to estimate its lifetime and possible future failures. Considering the need for improved asset management within large organizations, the use of thermal sensors becomes, even more, a viable option.

Since an infrared image has no engagement with the shape of inspected objects, but with relative representation of the temperature on the surface of such objects, the use of the infrared spectrum cameras for detecting or locating is not recommended. Factors like the scene's thermal uniformity and the difference of equipment's emissivities further complicate its use for this purpose. To contour this issue, hybrid systems are often used. Therefore, visible spectrum camera is used for recognizing objects of interest, while the infrared camera is responsible for its analysis.

For proper work, it is necessary that the objects of interest can be seen by both cameras. Considering this, it is essential to calibrate the system and to develop a methodology for determining the correspondence between the initially independent images [1].

Calibration can be defined as the calculation of intrinsic and extrinsic parameters of a camera. Such parameters are determined by means of rigs with known characteristics [2]. However, this process is relatively new for infrared cameras and requires some important adaptation points. One fundamental point is the use of a calibration rig that provides high thermal and visible contrast, allowing its distinction in both spectrums.

Pixel-by-Pixel alignment of two images can be performed once a set of common points is known. Using this set of points, it is possible to determine the difference in magnification and field of vision (FOV) besides the relative position between corresponding elements of this set. The calibration rig, which has known characteristics, may be used to obtain these points and to define the intersection of the two images.

The study of hybrid system's calibration process is one of the objectives of this paper. Furthermore, it is desired to perform the mapping between the images provided by the system using a specially developed calibration rig and to determine the metrological reliability of the proposed methodology. Sections II and III present a brief compilation of the main points related to individual and stereo calibration. Section IV shows an approach to deal with the design and construction of the mentioned calibration rig. Section $\mathrm{V}$ discusses the methodology for mapping the points of the Hybrid System's images. Section VI presents the steps for the metrological analysis of the methodology. Finally, the sections VII and VIII show the experimental results and the conclusions, respectively. 


\section{Calibration}

\subsection{Intrinsic and Extrinsic Parameters}

The extrinsic parameters describe how points in a world referenced coordinate system are mapped into a camera referenced coordinate system. After this mapping, it is necessary to translate the origin and change the metric system in order to obtain the values of these coordinates in pixels. For this, the intrinsic parameters of the camera must also be determined. Eq. 1 shows the relationship between a point in the world and its corresponding projection on an image.

$$
\left(\begin{array}{l}
v \\
v
\end{array}\right)=K\left[\begin{array}{ll}
R & T
\end{array}\right]\left(\begin{array}{l}
X \\
Y \\
Z \\
1
\end{array}\right)
$$

where $\mathrm{K}$ is the matrix containing the intrinsic parameters, $\mathrm{R}$ is the rotation matrix and $\mathrm{T}$ is the translation vector.

\subsection{Lens Distortions}

The calibration procedure still includes the determination of the radial and tangential distortions that are caused by the lens. The radial distortion, which represents the most significant distortions on commercial cameras, is due to the shape of the lens. This distortion is stronger in points further from the center of the image, ie, closer to the edges. The tangencial distortion is due to camera's assembling process, that causes the lens not be exactly parallel to the plane on which the image is formed. This fact causes physical processes called "thin prism" and / or "decentralization."

Despite other distortions present in imaging systems, the two types mentioned above are the most significant ones. The process of mathematically removing lens distortions, which is the main result of the calibration, is called rectification [2].

\section{Stereo Calibration}

Stereo calibration can be defined as determining the geometric relationship between two cameras in the space, in other words, the calculation of the rotation matrix and the translation vector between the two cameras. The information obtained through the calibration of each camera can be used to estimate the geometrical relationship between them. The rotation matrix and the translation vector between two cameras can be determined from Eq. 2 and Eq. 3, respectively.

$$
\begin{aligned}
& R=R_{\text {right }}\left(R_{\text {left }}\right)^{\mathrm{E}} \\
& t=t_{\text {right }}-R t_{\text {left }}
\end{aligned}
$$

$R_{Y}$ and $R_{\mathbb{l}}$ represent the rotation matrix from the camera positioned on the right and on the left, respectively. The vector $t_{r}$ is the translation vector of the right camera and ${ }^{t_{l}}$ is the translation vector of the left camera. In the end, ' $R$ ' and ' $t$ ' put the images on the same plane.

The process of aligning the lines of two images, called stereo rectification, is the final result of the stereo calibration. With this, it is possible to determine the correspondence between the images and generate a disparity map that relates corresponding points in each image.

The stereo calibration is quite interesting in applications where it is desired to obtain spatial information and is simplified by the use of identical cameras. However, the rectification of hybrid system's images, if not performed correctly, may distort the temperature information provided by the infrared camera. Furthermore, after the calibration process, it is still necessary to do a one dimensional search to determine the disparity between the images, which is not possible. As mentioned earlier, thermal images are not committed to the form of objects in a scene. In practice, the difference in resolution, magnification and FOV turn the stereo calibration process too difficult without prior image processing. Therefore, it would be interesting to develop another way to find the correlation between the pixels of a hybrid system's cameras.

\section{Calibration Rig}

As mentioned before, the calibration requires the use of a calibration rig with known features. In this process, each camera captures many views of the rig, being able to distinguish certain features invariant to rotation and displacement (e.g., corners). With this information, the parameters of interest for individual calibration are calculated and corresponding pair of points are determined for both cameras.

For calibrating visible and infrared cameras, it is necessary to build a calibration rig that can be seen simultaneously by both. The condition for a chessboard rig to be distinguishable in the infrared spectrum is that the same 
color squares present similar temperatures and different color squares present different temperatures. In addition, the temperature gradient between different color squares must be greater than the minimum temperature difference detectable by the infrared camera.

A proposal of a calibration rig for calibrating hybrid systems is presented in [1]. This proposal is based on the use of thermally insulated components, heated up to different temperatures to obtain the required minimum temperature gradient. Its results are quite interesting, but the construction has aspects that difficult its dissemination and use in noncontrolled environments. In the present paper, a different concept was used. For the calibration rig to show the required characteristics, materials with different emissivities have been used.

The infrared radiation received by a body is partly reflected and partly absorbed. Then a portion of absorbed energy is reemitted due to the increase of the molecules' kinetic energy. Emissivity is defined as the ratio between the radiation emitted by a surface and the radiation emitted by an ideal blackbody at the same temperature. Therefore, the portion of energy reemitted by the object is related to its emissivity. This parameter is dependent of the wavelength, the direction and especially the type of material [3].

The temperatures measured by infrared cameras depend on the material's emissivity, i.e., a higher emissivity material emit more radiation and thus will appear to have greater temperature than a material with lower emissivity. This fact is true even if both materials are actually at the same temperature. Therefore, it is expected that the necessary gradient for the calibration rig can be obtained by using materials with a reasonable difference of emissivity, without the need of artificial heating or thermal insulation. Fig. 1 shows the developed calibration targets.

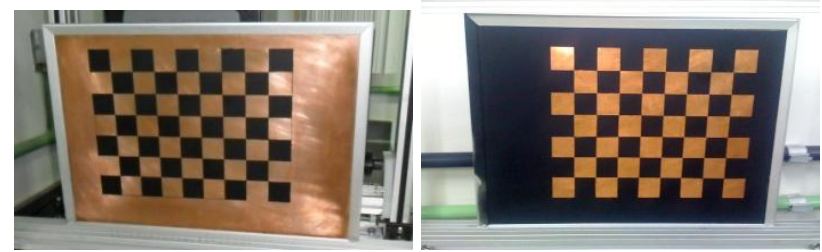

Fig. 1. Calibration Rigs

For the calibration rig on the left (rig 1), the background material is a copper plate, with emissivity about 0.09 . The squares were painted with high emissivity spray ink (approximately 0.98 ). The calibration rig on the right (rig 2) consists of a high emissivity background and copper squares.

It is important to note that the infrared camera requires adjusting the emissivity parameter for a correct measurement of temperature. This fact serves to ensure that measurement results are valid for all materials on the scene that present this emissivity value.

\section{Pixel-by-pixel alignment}

After removing the distortions of the images through the calibration process, it is necessary to determine the correspondence between the pixels of these images. This fact is critical to ensure that the segmentation of a visible image can be replicated in a thermal image, allowing the application of algorithms for automatic equipment evaluation. The images obtained by the cameras have different resolutions, magnifications and fields of view. Therefore, manipulations should be performed so that these aspects are compensated. These manipulations are described as follow.

\subsection{Magnification}

The use of lenses involves, besides the introduction of radial and tangential distortions, a magnification effect of the objects captured through it. To solve the problem of magnification difference introduced by the lens of each camera, one can use the concept of distance between adjacent corners. Fig. 2a illustrates this concept for a chessboard type calibration rig.
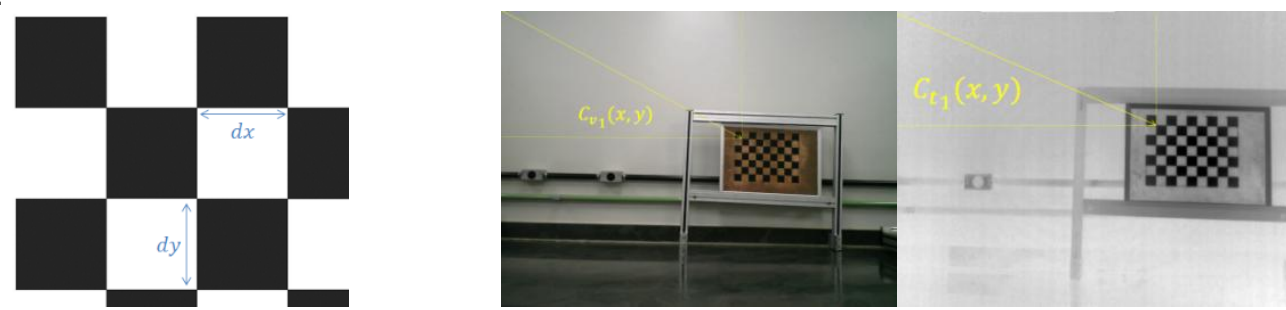

Fig. 2. a) Distance Between Adjacent Corners

b)Relative Position of a Correspondent Pair of Points

In Fig. $2 \mathrm{a}, \mathrm{dx}$ is the horizontal distance between two adjacent corners and $d y$ is the vertical distance between two other adjacent corners. As the calibration rig to be used is the same for both cameras, by knowing all distances 
$d x_{V I S}, d y_{V I S}$ (visible image) and $d x_{I R}, d y_{I R}$ (thermal image) it is plausible approximate the difference in horizontal and vertical magnification between the cameras by equations 4 and Error! Reference source not found.:

$$
\begin{aligned}
& M_{x}=\overline{d x_{V I S}} / \overline{d x_{I R}} \\
& M_{y}=d y_{V I S} / d y_{I R}
\end{aligned}
$$

Where $M_{x}$ is the horizontal magnification, $M_{y}$ the vertical magnification, $\overline{d x}$ and $\overline{d y}$ are the averages of all horizontal and vertical distances $d x$ e dy between adjacent corners.

After determining the horizontal and vertical magnification, one image is resized so that this effect is canceled. It is interesting to manipulate only the visible image, since resizing operations (interpolation, filtering and sampling) may misrepresent the physical meaning of the infrared image, creating an inconsistent temperature values or suppressing values of interest. Generally, the thermal image presents lower spatial resolution than the visible image, thus the objects would seem bigger on thermal image than on the visible one. In this case, the visible image needs to be augmented in order that the rig has the same size in the two images, satisfying the relationship $d x_{V I s_{\mathrm{II}}}=d x_{I R}$ e $d y_{V I s_{\mathrm{II}}}=d y_{I R}$.

\subsection{Relative Point Position}

After correcting the magnification problem, the detected corner's locations can be used to determine the relative position of the rig on the two images, as shown in Fig. 2b. Comparing the coordinates of each pair of corresponding corners, equation 6 gives the average difference between the position of a point on the new (augmented) visible image and the unaltered infrared image.

$$
\bar{C}(x, y)=\text { mean }\left(C_{V I S_{\mathrm{ri}}}\left(\frac{x}{M_{x}}, \frac{y}{M_{y}}\right)-C_{I R_{\mathrm{H}}}\left(x_{x} y\right)\right)
$$

Having the position difference between all known corresponding points, it is possible to determine the average point displacement $\bar{C}(x, y)$.

\subsection{Field of View}

Lastly, due to the different FOVs of each camera, there are regions of the images that are not common to both. This problem can be solved if these regions were not considered for the analysis, leaving only the parts that are seen by both cameras. Usually, the infrared camera's field of view is much smaller than the one of the visible camera. Therefore, knowing the relative position and size of the thermal image, it is possible to cut off non correlated areas in visible image, leaving virtually identical FOVs (except for the difference in horizontal and vertical angular position). Fig. 3 illustrates the difference between the fields of view of two cameras, highlighting the regions that are not entirely displayed on both images.

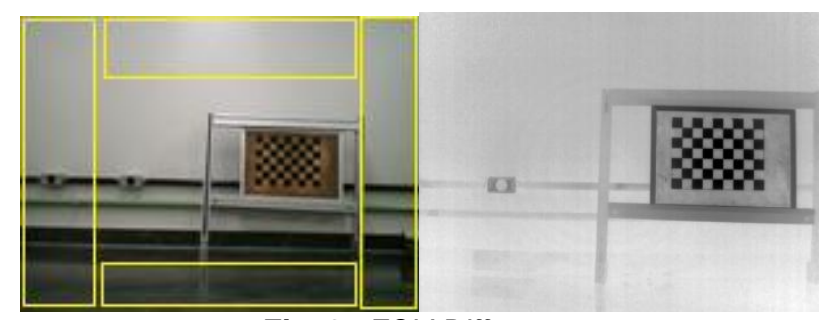

Fig. 3. FOV Difference

The methodology described in this section exclude the need to use the epipolar geometry and calculate the disparity between the images, which implies a simplification of the algorithms and results.

\section{Metrological Analyses}

The changes made to compensate FOV and magnification generate distortions that affect the exact correspondence between points on the visible image $P_{V I S}\left(x_{x} y\right)$ and the infrared image $P_{I R}\left(x_{v} y\right)$ (at temperature $\mathrm{T}\left(x_{v} y\right)$ ), as shown in Fig. 4a. In addition, there are also doubts about the exact positioning of points on the visible image after the corrections. This uncertainty comes from the own uncertainties of the data obtained by the calibration of the hybrid System, such as positioning, distance between cameras, target distance and distortions. Therefore, to a given point $P_{\text {VIS }}\left(x_{v} y\right)$ an offset coordinate error $\Delta \mathrm{x}$ and $\Delta \mathrm{y}$ can be assigned, relative to its corresponding point $P_{I R}\left(x_{v} y\right)$ in the infrared image, and also some uncertainty regarding its precise position, as shown in Fig. $4 \mathrm{~b}$. Consequently, the 
$P_{\text {VIS }}(x, y)$ point is inserted within the region of uncertainty defined by the rectangle of $2 u_{x}$ and $2 u_{y}$ size, which are the coordinates uncertainties of $P_{\text {VIS }}(x, y)$ in the horizontal and vertical directions, respectively.
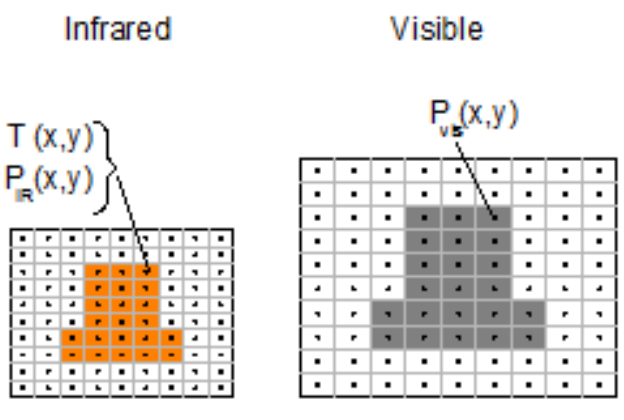

a)

Fig. 4. a) FOV and Magnification Correction

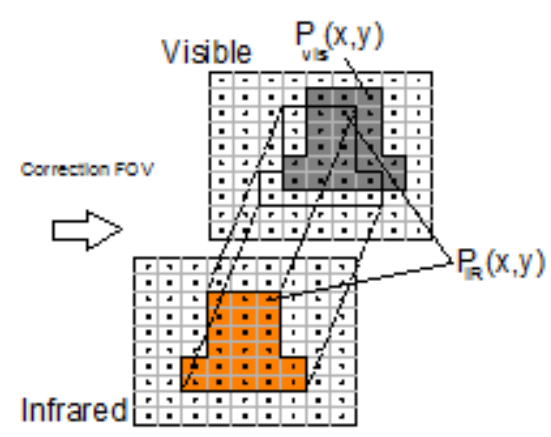

b) Correspondence Between $R_{V I S}(x, y)$ and $P_{I R}(x, y)$

It is important to note that associate $P_{V I S}(x, y)$ to its corresponding point $P_{I R}\left(x_{v} y\right)$ in the infrared image, means associate the temperature, $T\left(x_{x} y\right)$ (of $P_{I R}\left(x_{x} y\right)$ ) to the point $P_{V I S}(x, y)$. However, if the location of $P_{V I S}(x, y)$ cannot be determined exactly, the temperature $T\left(x_{v} y\right)$ cannot be associated with $P_{V I S}\left(x_{v} y\right)$ without doubt. Fig. 5a shows the situation where $P_{V I S}(x, y)$ is in uncertainty zone which includes the surface of the object of interest and a background. Hence, the temperature $T\left(x_{v} y\right)$ associated with $P_{V I S}(x, y)$, may be the temperature of the object or the background. It is, therefore, necessary to define a measurement area where a temperature $T^{\prime}\left(x_{v} y\right)$, associated with $P_{\text {VIS }}^{\prime}\left(x_{v} y\right)$, can safely be attributed to the surface of the object whose temperature is desired. Fig. $5 \mathrm{~b}$ illustrates a measurement area smaller than the object's edges by $u_{x}$ and $u_{y}$ pixels in the horizontal and vertical directions, respectively. The uncertainties and errors associated with $P_{V I S}^{\prime}\left(x_{v} y\right)$ must be reduced so that the measurement area is satisfactory to a defined purpose.

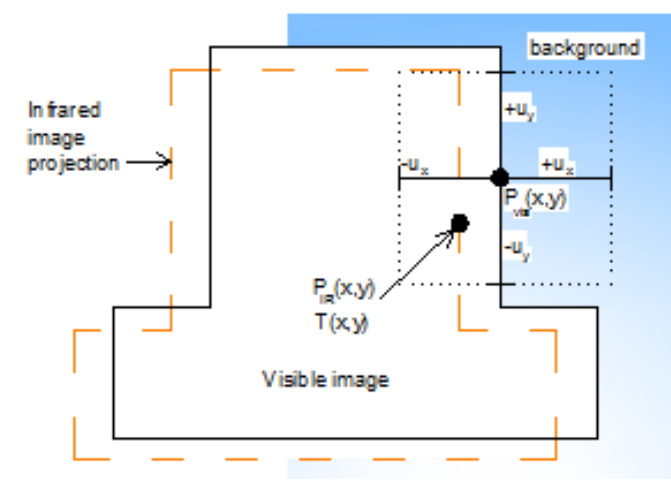

Fig. 5. a) Temperature Measurement of an Uncertainty Point

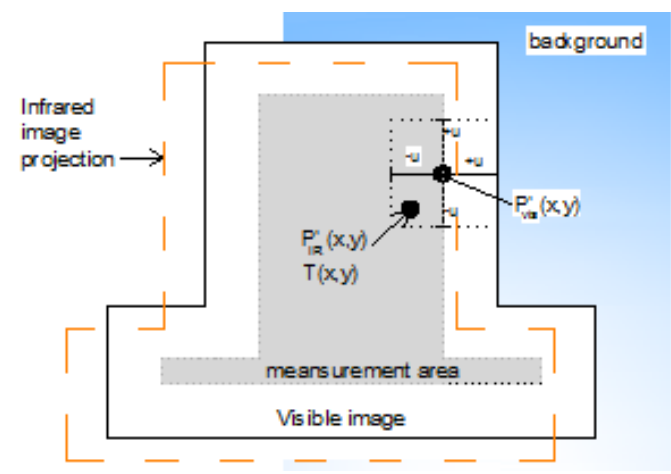

b) Measurement Area

\section{Experimental results}

To verify the functionality of the developed rigs and demonstrate the concepts discussed above, the pixel-bypixel alignment of a hybrid system consisting of a Microsoft LifeCam VX7000 camera on the left of a FLIR SC660 infrared camera was performed. The algorithms were implemented using the OpenCV 2.2 [4] and the Camera Calibration Toolbox for MATLAB [5]. MATLAB® [6] was also used for metrological analysis of the methodology. Fig. 6 illustrates the main functions of the developed algorithm. 


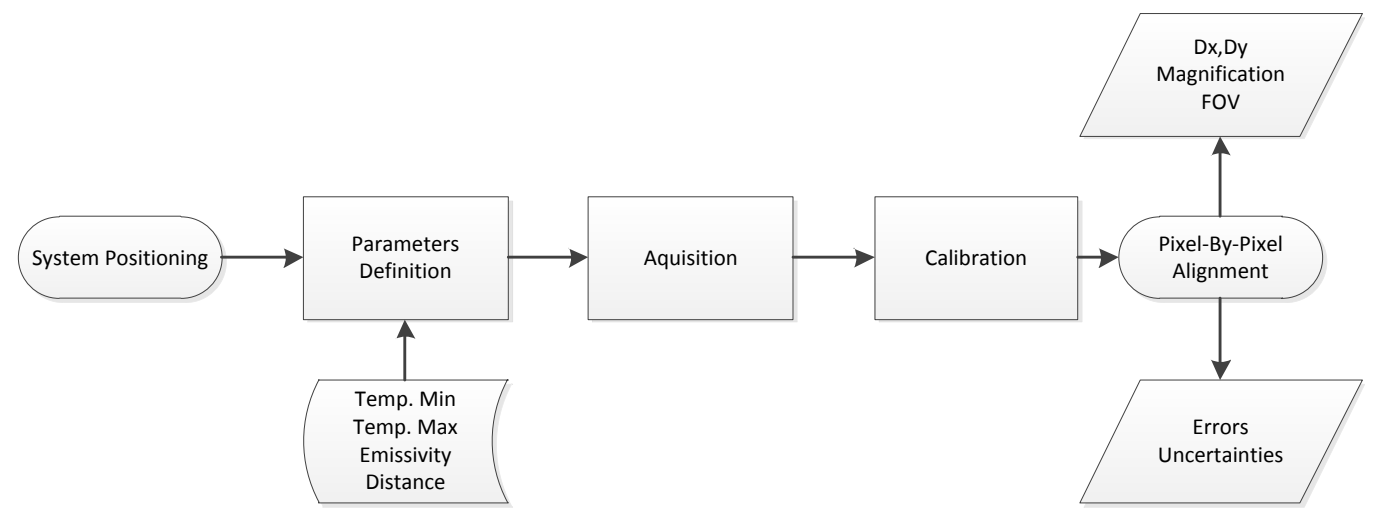

Fig. 6. Pixel-by-pixel Alignment Algorithm

In the first case, at the laboratory, the images were captured with the rigs at room temperature of about $24^{\circ} \mathrm{C}$ (Fig. 7). In the second case, the calibration rigs were artificially heated up to $30^{\circ} \mathrm{C}$ (Fig. 8). At both first and second cases, the rigs were at a perpendicular distance of $1.4 \mathrm{~m}$ from the cameras. In the third case, the targets were exposed to sunlight for a period of 5, 10 and 20 minutes and acquisitions have been made to each of these situations (Fig. 9 and Fig. 10). The environment temperature was $26.5^{\circ} \mathrm{C}$ and relative humidity was $59 \%$.

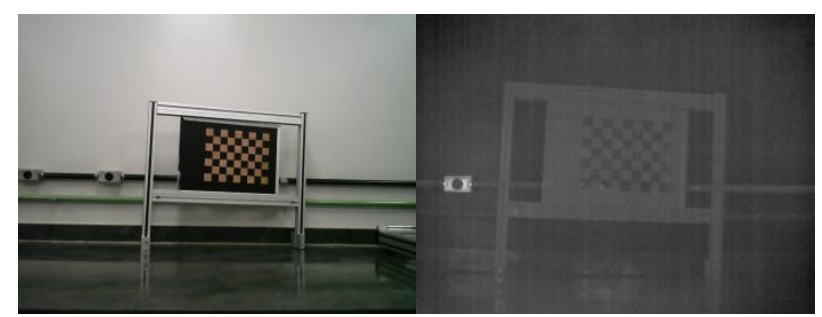

Fig. 7. Case 1: Laboratory's Room Temperature
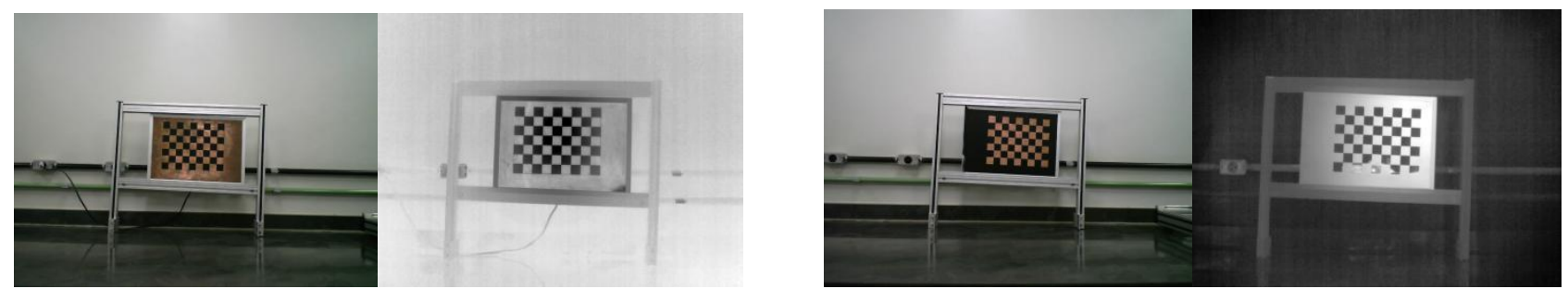

Fig. 8. Case 2: Artificial Heating at the Laboratory

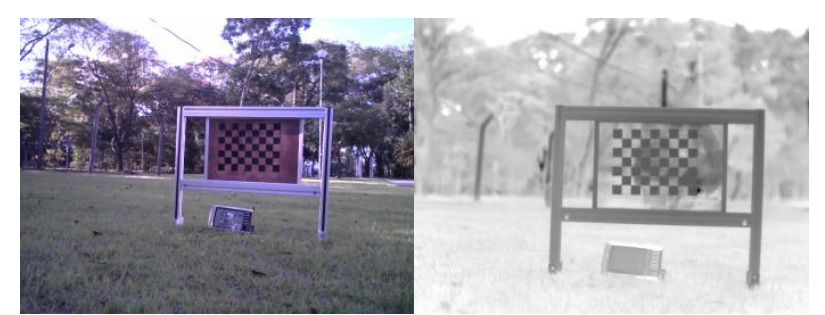

Fig. 9. Case 3: External Environment - Rig 1 

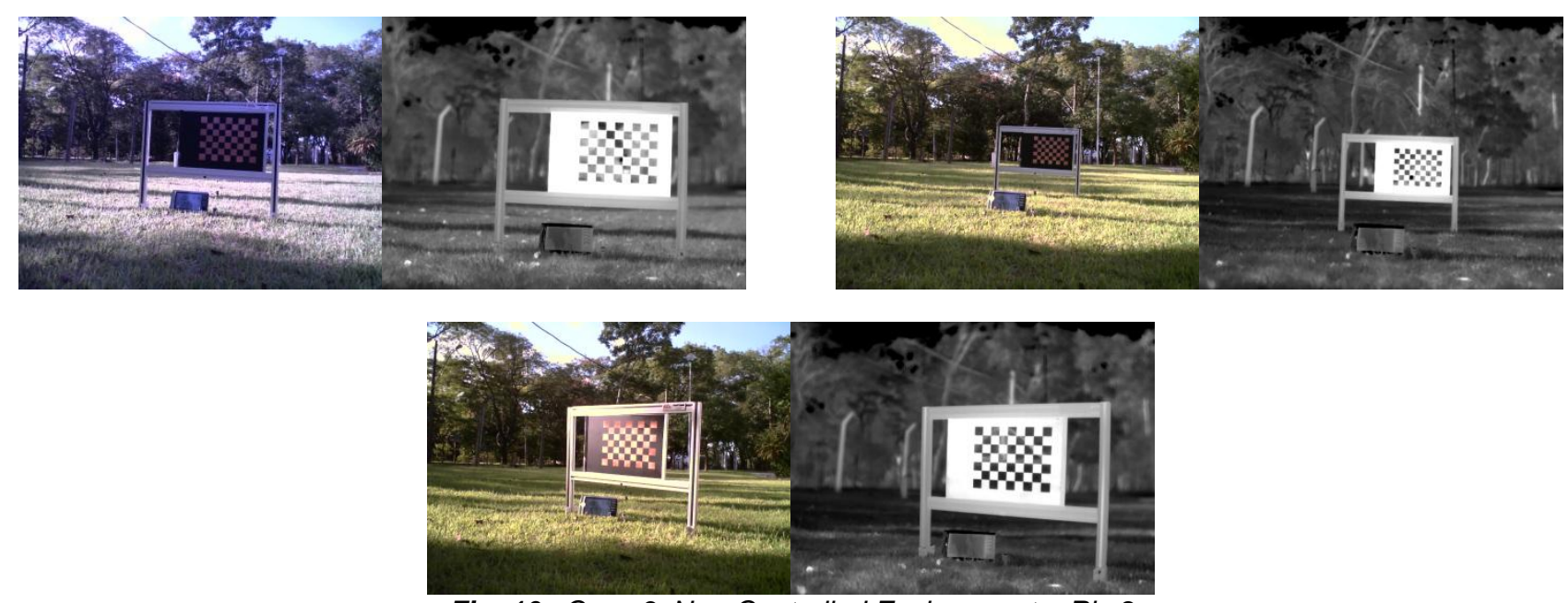

Fig. 10. Case 3: Non-Controlled Environment-Rig 2

The temperature gradient between the copper squares and the high emissivity background in the first case was only $0.3^{\circ} \mathrm{C}$, however, it was still possible to detect the corners of rig 2 . In all other cases it was possible to perform the detection of the corners for both rigs. It is worth noting that rig 1 presented a significant amount of reflection on outdoor experiments due to the copper background, which made corner detection more difficult. During the third case, the rigs reached temperatures above $37^{\circ} \mathrm{C}$. In Fig. 10 targets were at $1.5 \mathrm{~m}, 2.5 \mathrm{~m}$ and $1.5 \mathrm{~m}$, respectively.

After corner detection, their coordinates are stored and supplied to the algorithm that will estimate the intrinsic and extrinsic parameters and remove the images' distortions. From the extrinsic parameters is possible to determine the relative position between the two cameras according to Eq. 2 and 3. Figure 11 illustrates the results of corner detection for infrared images.

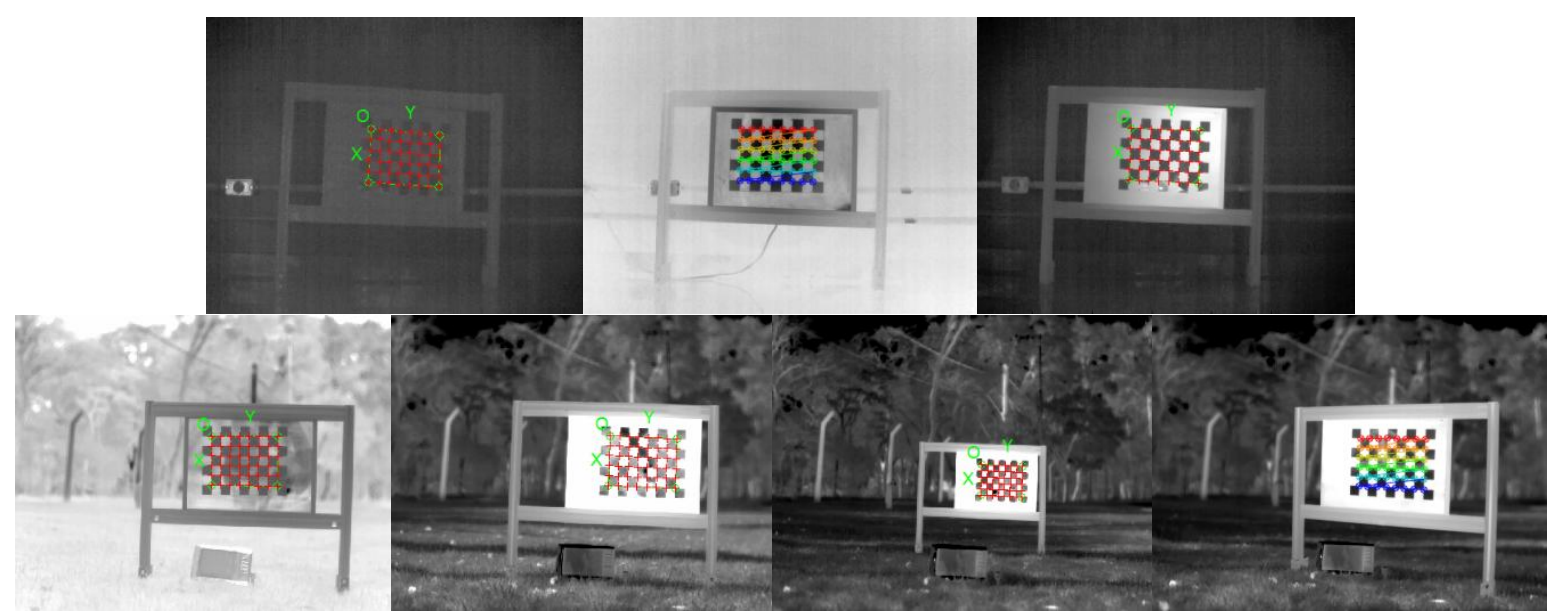

Fig. 11. Corner detection

The last step is the execution of the pixel-by-pixel alignment algorithm. The outputs of this algorithm are the average values of horizontal and vertical magnifications and the average relative position of corresponding points. Besides that, the visible images are adjusted to become much closer to the corresponding thermal ones. The results of applying the algorithm for each case are shown in Fig. 12 to figureFig. 15.

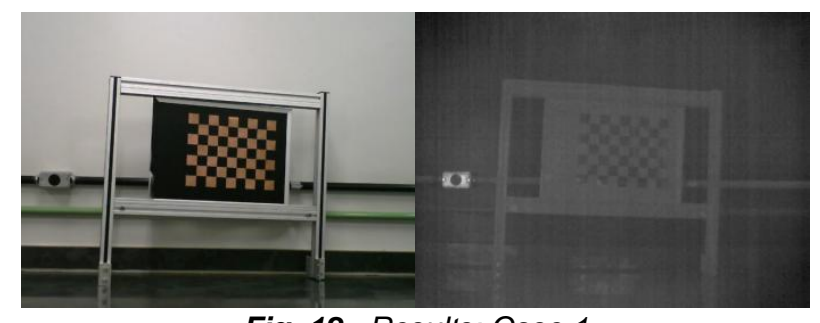

Fig. 12. Results: Case 1 

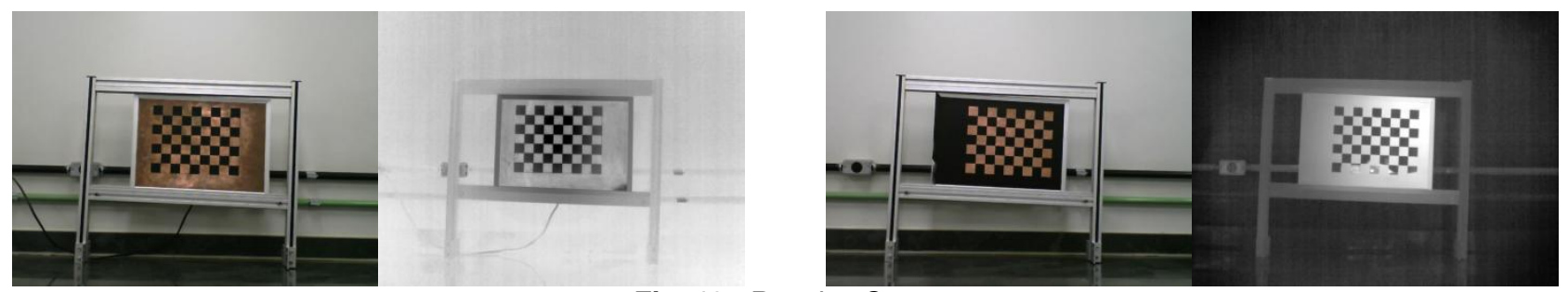

Fig. 13. Results: Case 2

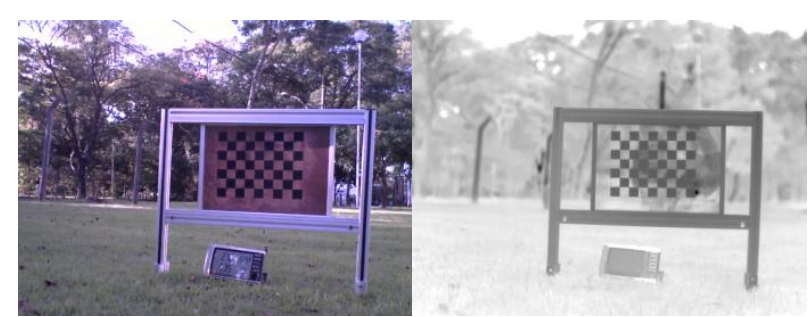

Fig. 14. Results: Case 3 - Rig 1

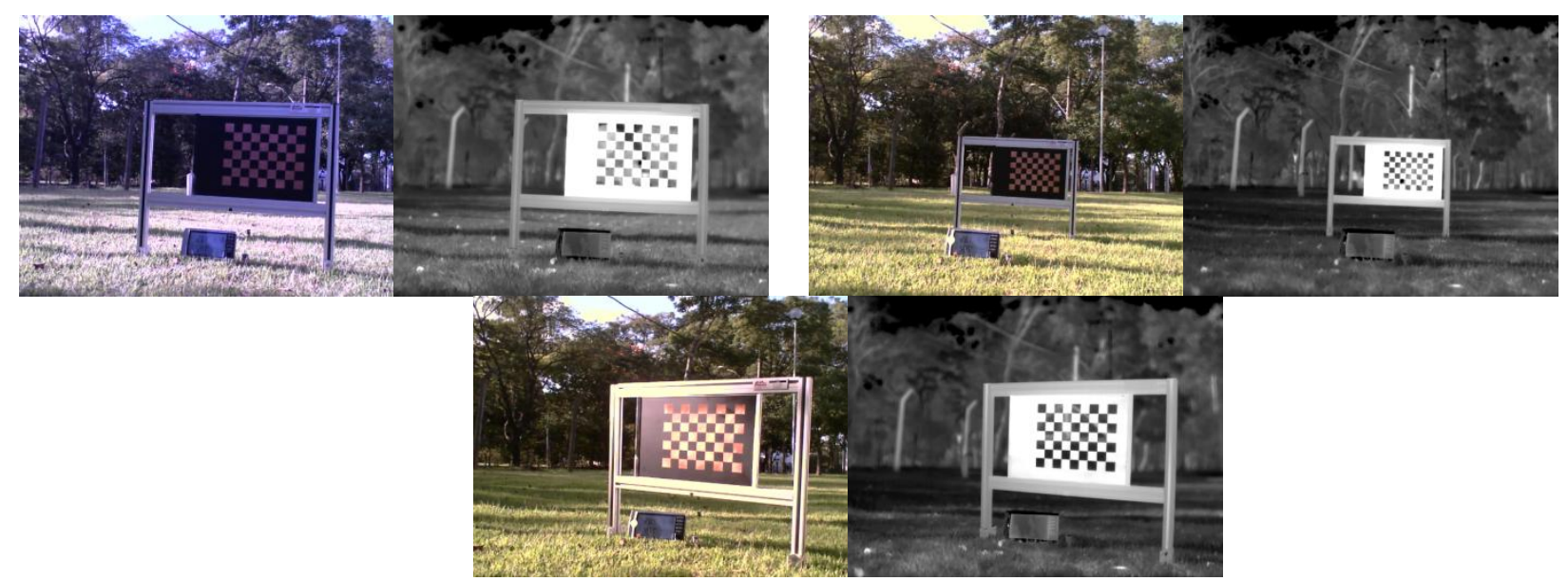

Fig. 15. Results: Case 3 - Rig 2

Table 1 shows the average errors and their uncertainties, besides the uncertainties of the average coordinates of each calibration for cases 1 to 3, shown in Fig. 12 to Fig. 15. The uncertainties of the calibration points were calculated by Monte Carlo method [7], considering the standard deviations provided by the Camera Calibration Toolbox and dispersion occurred in the FOV compensation and magnification correction algorithms.

Table 1. Errors and uncertainties

\begin{tabular}{|c|c|c|c|c|}
\hline Case - Rig & Environment & Coordinate & $\stackrel{\text { Error }}{\Delta x}, \underset{\Delta y}{\text { [pixels] }}$ & $\begin{array}{c}\text { Uncertainty } \\
\pm \vec{u}_{x}, \pm \overrightarrow{u_{y}} \text { [pixels] }\end{array}$ \\
\hline \multirow{2}{*}{ Case 1 - Rig 2} & \multirow{2}{*}{ Laboratory } & $x$ & $2.51 \pm 1.54$ & \pm 5.81 \\
\hline & & $y$ & $0.73 \pm 0.91$ & \pm 0.89 \\
\hline \multirow{2}{*}{ Case 2 - Rig 2} & \multirow{2}{*}{ Laboratory } & $x$ & $1.40 \pm 0.61$ & \pm 5.57 \\
\hline & & $y$ & $0.59 \pm 0.76$ & \pm 0.89 \\
\hline \multirow{2}{*}{ Case 2 - Rig 1} & \multirow{2}{*}{ Laboratory } & $x$ & $1.52 \pm 0.38$ & \pm 1.45 \\
\hline & & $y$ & $1.49 \pm 0.77$ & \pm 1.83 \\
\hline \multirow{2}{*}{ Case 3 - Rig 1} & \multirow{2}{*}{ Outdoors } & $x$ & $0.65 \pm 0.87$ & \pm 1.10 \\
\hline & & y & $0.48 \pm 0.69$ & \pm 1.00 \\
\hline \multirow{2}{*}{ Case 3 - Rig 2} & \multirow{2}{*}{ Outdoors } & $x$ & $0.45 \pm 0.57$ & \pm 1.83 \\
\hline & & $\mathrm{y}$ & $0.33 \pm 0.41$ & \pm 1.18 \\
\hline
\end{tabular}



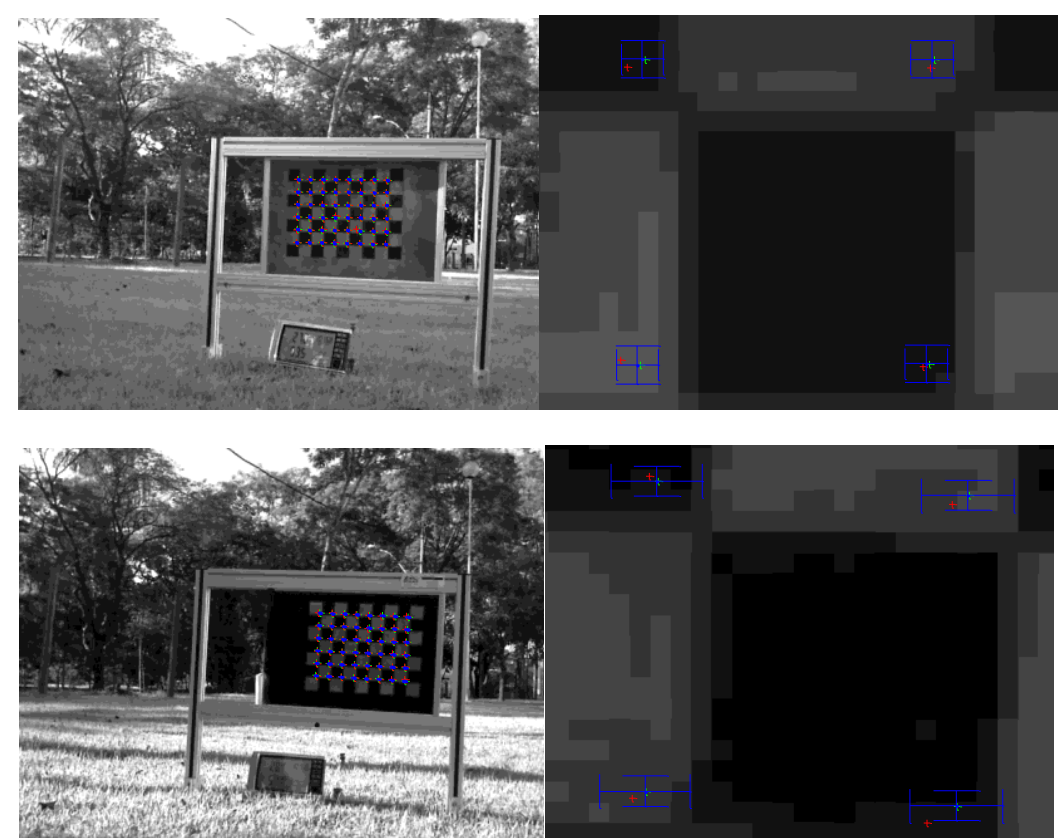

Fig. 16. Calibration Points of Rigs 1 and 2

\section{Conclusions}

The calibration of hybrid systems may be performed using a calibration rig with particular characteristics. On this subject it is important to emphasize some points: A good resolution facilitates corner detection and allows greater distances between the cameras and the rig. Moreover, it is better that the rig does not stay too close to the system, since the infrared camera does not have a very wide field of view and may require certain distance to allow good visualization.

A very promising result of this paper was the developed calibration rig. The behavior of the rig in uncontrolled environment exceeded project expectations, proving to be a very viable tool. Besides being low cost, its construction is quite simple, allowing a wide use in applications that need to perform infrared camera calibration. Rig 1 presented great susceptibility to reflection, but this was significantly reduced with rig 2, since the copper area (low emissivity) was minimized.

There are points that must be observed for obtaining good thermal images of the rigs, such as reducing sources that may cause reflection at the low emissivity area and removing objects with extreme temperatures from the scene, if possible. It is suggested the correct choice of infrared image's upper and lower temperature limits, in order to achieve the highest possible contrast and benefit corner detection. Determining the high emissivity area temperature and working in a range close to it is a good strategy for an initial setting of this limits. In the low emissivity regions, the reflection of objects with temperatures close to the one presented by the high emissivity region should be avoided since it hinders rig visualization.

It is recommended thee used of image post-processing operations in order to facilitate corner location. Thresholding can be utilized for best results, for example. Another possibility is morphological operation for noise elimination and closure of small holes. However, one must carefully select the threshold for binarization and choose the characteristics of structural elements.

The correspondence quality between hybrid system's images is essential so that effective temperature measurement of identified objects and its distinction from the background can be assured. In this context, the developed pixel-by-pixel alignment method is a viable solution for equipment's temperature measurement. Results show that the proposed methodology is valid, with low rate errors and low uncertainty in altered visible image's point position. The calibration rig presented excellent results, especially in non-controlled environments, as shown by the results listed in

Table 1. In future works, further investigation should be conducted to define how uncertainties in a given point position on the visible image affects the uncertainty of its associated with the temperature.

The relative positioning between system's cameras and their distance from the rig are important factors and should always be observed. Increasing the number of images used as input to the alignment method reduces results standard deviation and increases the method's reliability. Therefore, one must determine a balanced relationship between the number of images to be used and the computational effort required to analyze the related data.

The advance and dissemination of hybrid systems can bring many benefits if these systems are correctly used. Certain that calibration and pixel-by-pixel alignment are crucial for a wider applicability of such systems, the study of these processes and the development of tools for algorithm optimization become very promising within the area of infrared image and computer vision. 


\section{REFERENCES}

[1] St-Laurent L., Prévost D., Maldague X. "Fast and accurate calibration-based thermal/colour sensors registration", Proceedings of 10th Quantitative InfraRed Thermography conference, paper QIRT2010-126 Québec (Canada), 2010.

[2] Bradski, G., \& Kaehler, A. "Learning OpenCV - Computer Vision with the OpenCV Library". O’Reilly Media, 2008.

[3] Incropera, F. P., \& Witt, D. P. "Fundamentals of Heat and Mass Transfer". John Wiley \& Sons, 2006.

[4] OpenCVWiki,2012. Accessed on April 17, 2012. Available at http://opencv.willowgarage.com/wiki/.

[5] Bouguet, Jean-Yves "Camera Calibration Toolbox for Matlab",2010. Accessed on April 30, 2012. Available at http://www.vision.caltech.edu/bouguetj/calib_doc/index.html.

[6] MathWorks, MATLAB, 2012. Available at http://www.mathworks.com/products/matlab/.

[7] Teixeira, G.G.D., Gomes Jr, S.C., Ferreira, E.H.B., Diniz, H.E.P., Andrade, R.M., "Avaliação de Incerteza de Resultado de Medição em Termografia Infravermelha pelos Métodos Gum e Monte Carlo, aplicada ao diagnóstico de conexões elétricas”, X Congresso Ibero-Americano de Engenharia Mecânica - Cibem 10, Porto (Portugal), 2011. 